\title{
La mécanique des fluides et les études sur maquettes au service des procédés industriels et des problèmes de pollution de l'environnement
}

\author{
Max Milhe \\ Responsable des études d'environnement atmosphérique et de procédés industriels \\ ACB-C.E.R.G., Groupe GEC ALSTHOM \\ Rue Lavoisier - 38000 Le Pont de Claix - 76.40.90.40
}

\section{Introduction}

Les écoulements rencontrés dans les procédés industriels, que ce soit de liquides ou de gaz, dans les circuits et leurs composants, dans les machines tournantes ou alternatives, dans les dispositifs multiples assurant les fonctions de distribution, de répartition, de mélange, de tranquillisation de fluides..., peuvent évoluer dans une gamme de vitesses très étendue: de quelques millimètres par seconde à plusieurs dizaines, voire centaines de mètres par seconde.

Dans ces écoulements peuvent intervenir des différences de densité et des différences de température entre fluides en présence, des mélanges polyphasiques de type liquidegaz (brouillards, émulsions), liquide-solide et gaz-solide (éléments en suspension), souvent une combinaison de ces différents phénomènes.

On conçoit ainsi que le problème du concepteur et de l'analyse des fonctionnements et des procédés ne soient pas simples: les simulations sur maquettes physiques à échelle réduite, voire quelquefois agrandie en fonction de la dimension du phénomène à analyser, associées aux outils informatiques, sont alors les outils utilisés par le mécanicien des fluides pour faciliter la compréhension de l'interaction des différents paramètres, aider au dimensionnement des installations concernées et éviter les accidents de fonctionnement parfois graves pour l'environnement, les personnes et les installations.

Les sujets évoqués ci-après montrent, parmi le large éventail des implications de la Mécanique des Fluides, quelques études types qui sont abordées en laboratoire au C.E.R.G. par modélisation sur maquettes, par exemple pour résoudre les problèmes de mélanges de fluides visqueux avec particules en suspension ou les études de pollution atmosphérique et d'environnement.

\section{L'étude sur maquette du mélange des compo- sants d'un pot de peinture pour l'industrie automobile}

\subsection{Nature du problème}

On s'intéresse ici au cas des pots de peinture utilisés dans l'industrie automobile : soit en fin de chaînes automatisées de peinture lorsque des retouches s'avèrent nécessaires, (la nuance de la couleur pouvant lentement varier entre le début et la fin d'une série), soit dans les centres de réparation de véhicules accidentés lorsqu'il s'agit de retrouver l'exacte nuance d'un coloris, corrections faites de la variation de la couleur avec l'âge du véhicule, le vieillissement variant lui-même avec l'ensoleillement (la correction sera différente dans le Nord de la France et dans le Sud, en Europe ou au Japon, etc...).

L'obtention d'une nuance donnée ou souhaitée (il en existe près de 50000 pour la France, 200000 pour l'Europe et 500000 dans le monde), résulte du mélange de couleurs de base ou "primaires" dans des proportions appropriées, la précision pondérale nécessaire à l'obtention de la nuance étant de l'ordre du 1/10 de gramme pour la réalisation de pots de l'ordre de quelques kilogrammes (en général 1 à $5 \mathrm{~kg}$ ).

Il convient donc, avant même d'obtenir la nuance souhaitée aux précisions requises, que les peintures "primaires » soient parfaitement homogènes : ce qui n'est évidemment pas le cas quand les pots issus de l'unité de fabrication ont pu rester stockés quelques semaines, voire quelques mois, avant une utilisation.

Ce stockage prolongé provoque par exemple une décantation sensible des pigments dans le vernis et le solvant, le phénomène étant aggravé avec les peintures métallisées ou mica : c'est l'étude expérimentale du procédé d'homogénéisation des pots de peinture "primaire » qui est présentée ci-après.

\subsection{Etude expérimentale}

\subsubsection{Le dispositif expérimental}

Le matériel utilisé est un prototype industriel, à l'échelle géométrique $1 / 1$, avec des adaptations qui permettent les mesures et les observations :

- système d'entraînement du mélangeur avec une précision de $1 \%$ sur la vitesse de rotation, avec une échelle de vitesse Nmodèle/Nréel de 1/1,

- réalisation d'un pot fictif en matériau transparent, à l'échelle $1 / 1$ des dimensions internes mais avec des faces 
externes planes pour éviter la déviation parasite des observations et des plans lumineux aux interfaces air-plexiglass par différence d'indice de réfraction,

- le montage expérimental permet à la fois des observations frontales et par le fond transparent du pot, à travers un miroir à $45^{\circ}$ (fig. I),

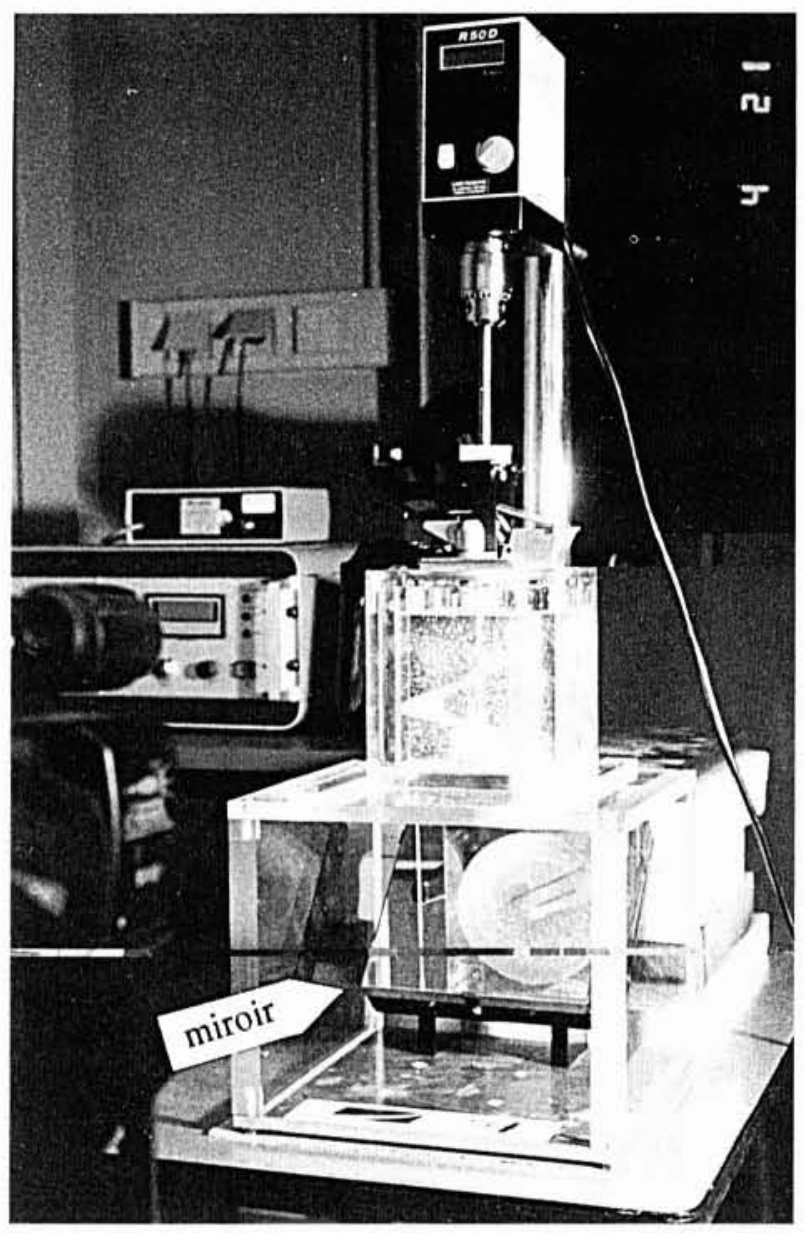

1. Moyen d'essais expérimental.

- les pales étudiées sont à l'échelle $1 / 1$ des dimensions réelles, en tenant compte des techniques de fabrication, des facilités de mise en place et autres contraintes,

— un système « dérotateur " à prisme tournant permet par procédé optique de rendre. fictivement, l'observateur et un appareil photo ou vidéo solidaires de l'axe de rotation de l'agitateur et d'observer ainsi, en continu, les écoulements autour de la pale, en faisant abstraction du mouvement général de rotation de celle-ci,

- la viscosité des peintures analysées étant de l'ordre de 800 à $1000 \mathrm{CSt}$, il a été utilisé un produit de viscosité identique mais transparent, à base de glycérol, pour permettre les visualisations au moyen de particules mises en suspension dans le fluide, soit une échelle $1 / 1$ des viscosités, le glycérol et la peinture ayant par ailleurs sensiblement la même densité $(1,1-1,2)$.

\subsubsection{Nature des résultats obtenus}

\subsubsection{Visualisation globale}

Les visualisations ont été réalisées au moyen de paillettes d'aluminium réfléchissantes, en suspension dans le fluide, ou au moyen de particules de PVC de quelques dixièmes de millimètre de diamètre.

L'association de particules de densité, de dimension et de couleur différentes, plus lourdes (sable fin, densité 2,65) et plus légères (PVC, densité de l'ordre de 1) a été également utilisée pour différencier le comportement de la phase légère (vernis-solvant) et de la phase lourde (pigment), tous les mouvements étant suivis par caméra vidéo, pour analyse détaillée et chronométrée.

\subsubsection{Visualisation par tranche lumineuse à balayage laser}

Cette technique permet, par rapport à la tranche lumineuse classique, une description du champ des vitesses dans le plan éclairé, chaque particule, sur un cliché de temps de pose assez long, étant éclairée à différentes positions sur sa trajectoire, à des intervalles de temps égaux dépendant de la fréquence de balayage du rayon laser qui assure l'éclairage du plan visualisé : les figures 2 et 3 montrent des exemples de visualisation par tranche à balayage où les clichés définissent à la fois des schémas d'écoulement et des champs de vitesse.

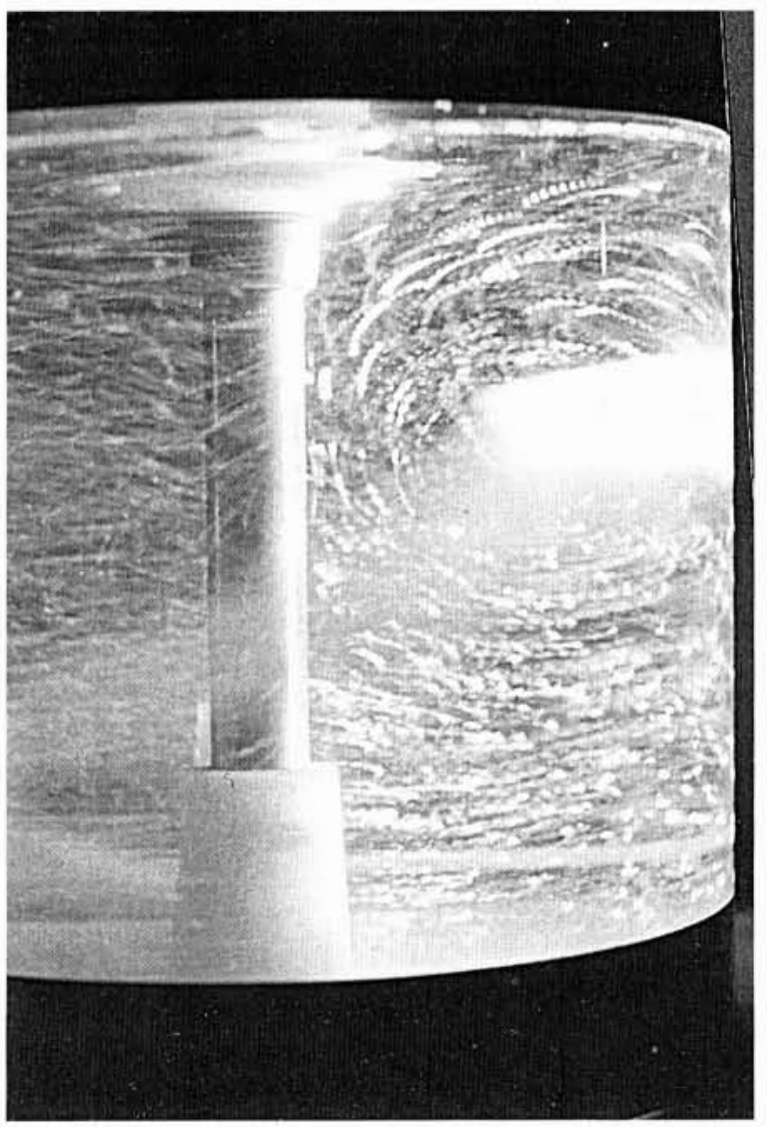

2. Visualisation des écoulements dans un plan diamétral. 


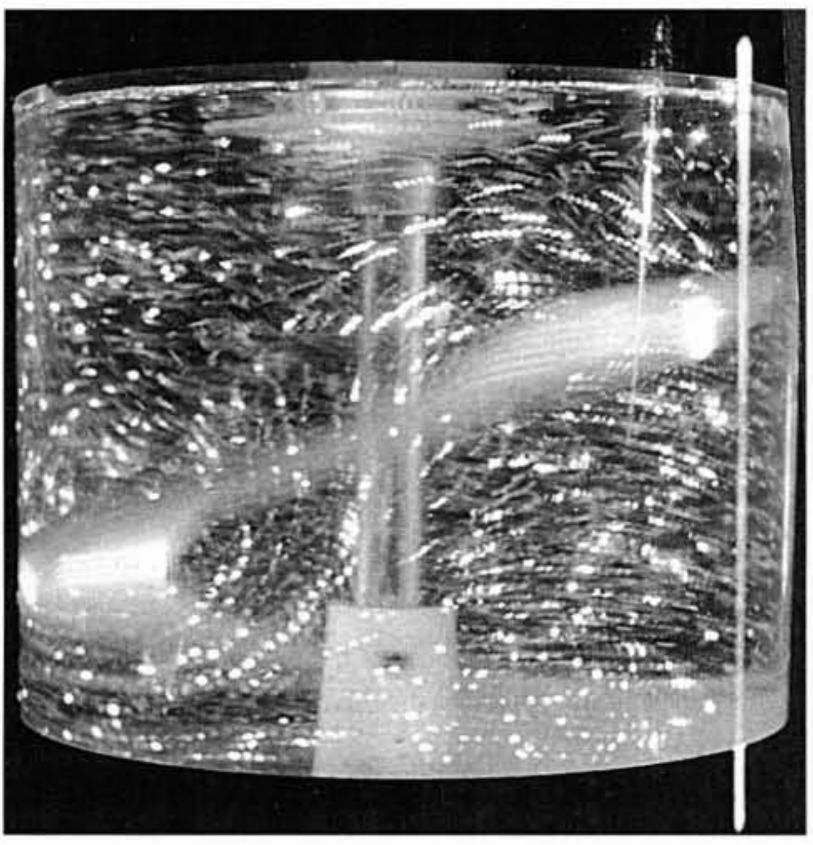

3. Visualisation des écoulements en avant du plan diamétral.

2.2.2.3. Contrôle final de l'efficacité de mélange par sédimométrie et analyse de couleur

Pour finaliser l'analyse de l'efficacité du mélange, deux types de contrôle peuvent in fine être réalisés sur peinture réelle :

- la détection au sédimomètre des éventuelles stratifications résiduelles dans le pot. Ce dispositif permet la mesure de l'effort de pénétration d'un plongeur dans la peinture, effort qui varie avec la profondeur de pénétration et avec la nature des constituants rencontrés ;

- le rendu de couleur obtenu avec différents échantillons de peinture du même pot.

\section{La similitude sur maquettes en analogie hy- draulique appliquée à l'étude des problèmes d'environnement}

\subsection{Introduction}

L'étude des phénomènes de diffusion des produits polluants dans l'atmosphère fait presque toujours intervenir l'influence des différences de densités entre des rejets et I'atmosphère ou dans l'atmosphère elle-même, que cette différence soit due à des écarts de température ou à des différences de nature de gaz.

Hormis quelques cas relativement rares où ces problèmes peuruent être abordés par le calcul, on a la plupart du temps recours aux modèles à analogie physique, en particulier la soufflerie à air et le tunnel hydrodynamique, pour tenir compte des particularités des différents problèmes étudiés : présence de relief, interaction de sillages de bâtiments ou d'obstacles, sources multiples, ...
La réalisation d'études théoriques préalables et une longue pratique des problèmes de diffusion atmosphérique ont montré que la modélisation hydraulique présentait certains avantages sur les souffleries conventionnelles, en particulier pour l'étude des effets thermiques et des vents faibles générateurs de mauvaise dispersion.

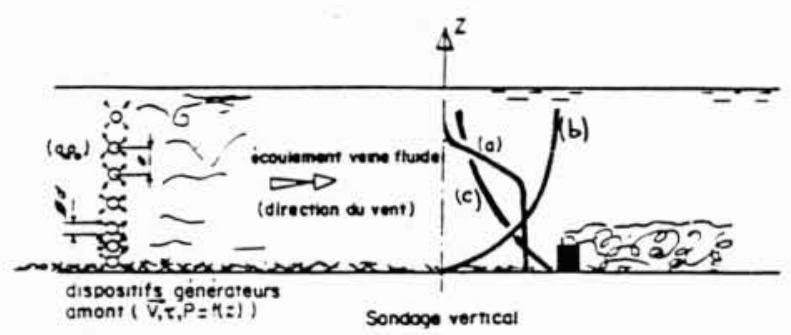

4. Procédure expérimentale, réalisation et mesure des profils de densité (a), de vitesse (b), de turbulence (c).

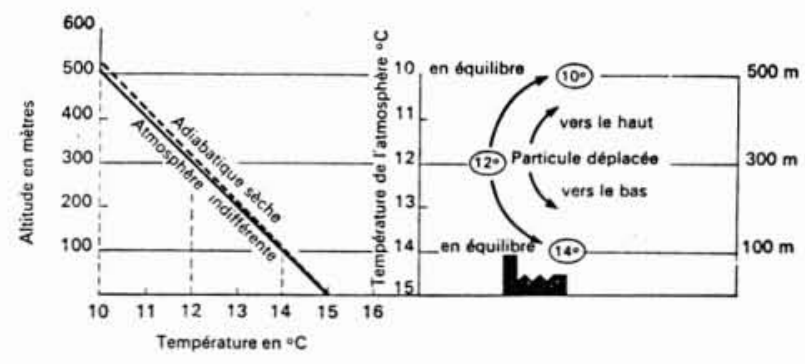

a - atmosphère adiabatique

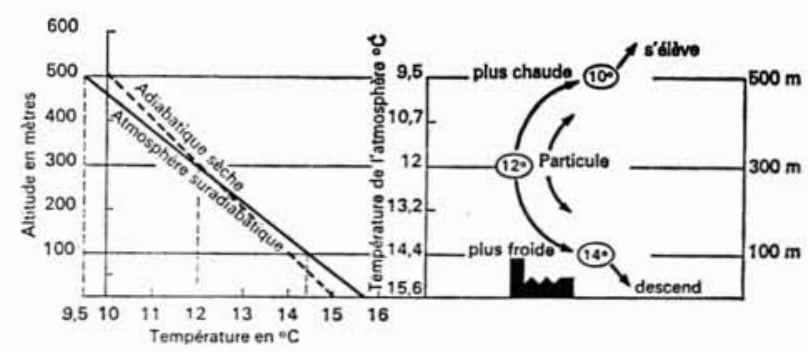

b - atmosphère instable

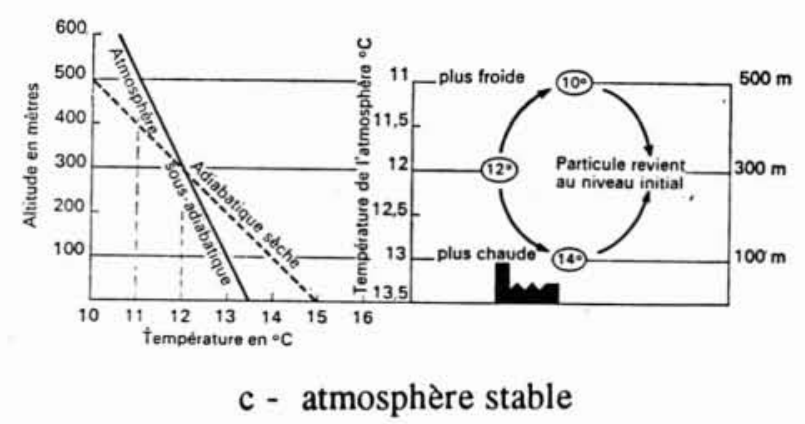

5. Profils thermiques verticaux et stabilité. 


\subsection{La modélisation de l'atmosphère en similitude hydrau- lique}

\subsubsection{Profils verticaux de température}

On sait que dans une atmosphère classique dite également " neutre " ou " adiabatique », il existe une diminution de la température de l'air en fonction de l'altitude, en moyenne d'un degré par cent mètres (fig. $5 a$ ) : c'est un phénomène bien connu en montagne et en navigation aérienne.

Dans ce schéma (a), une masse d'air, par exemple brutalement déplacée vers le haut par les mouvements de l'atmosphère, se détend et donc se refroidit, à raison également d'environ un degré par cent mètres de déplacement d'altitude, et se retrouve ainsi toujours, quels que soient son niveau de départ et son niveau d'arrivée, à la même température et à la même densité que les masses d'air voisines : elle reste alors en équilibre indifférent (fig. $5 a$ )

\section{a - inversion d'altitude à $300 \mathrm{~m}$}

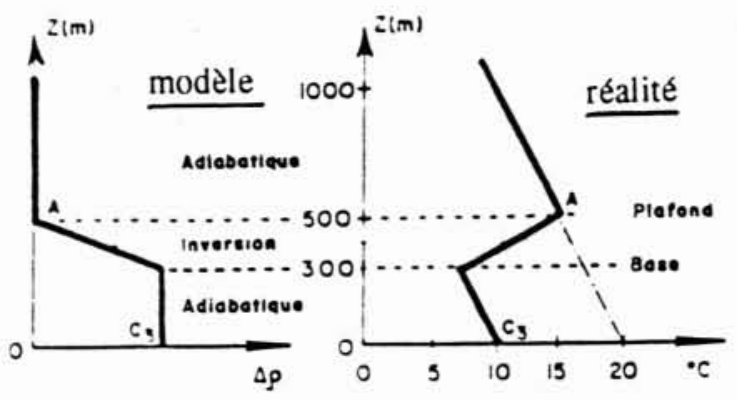

$\mathrm{b}$ - isothermie jusqu'à $500 \mathrm{~m}$

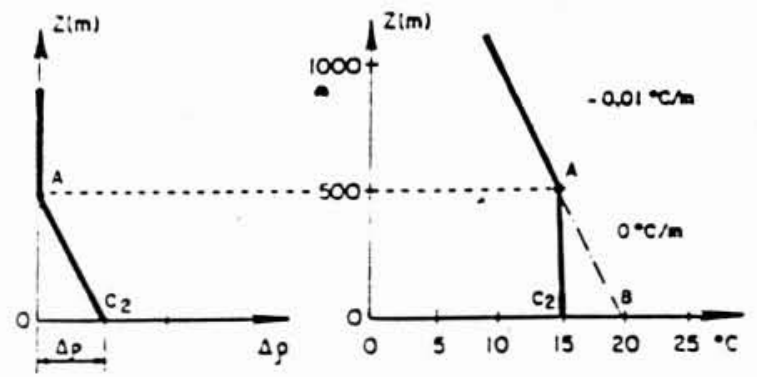

\section{c - inversion au sol}

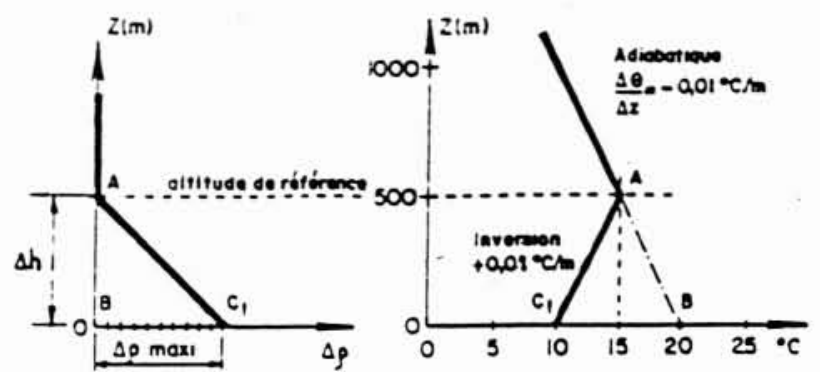

6. Simulation hydraulique des différents profils thermiques atmosphériques. et n'a pas tendance à s'écarter de sa nouvelle position d'équilibre comme ce serait le cas en atmosphère suradiabatique instable (fig. $5 \mathrm{~b}$ ), ou à y revenir naturellement dans le cas d'atmosphère stable (fig. $5 c$ ).

La veine d'eau du modèle expérimental où les volumes fluides ont partout la même densité (eau pure sur toute la hauteur, en équilibre indifférent) est donc bien représentative de l'atmosphère " adiabatique " standard, ou « neutre ». L'analogie hydraulique permet également la simulation des situations d'atmosphères stables et des inversions de température par la réalisation de gradients verticaux de densités sur la maquette, au moyen de saumure de densités variables, analogues à des profils de températures (fig. 6). Peuvent ainsi être réalisées, par le choix des épaisseurs de couches stables et de leur densité, aux échelles appropriées: des inversions d'altitude (fig. 6a), des situations de faible stabilité (isothermie, fig. $6 b$ ) ou de forte stabilité (fig. 6c).

\subsubsection{Profils de vitesses de vent et de turbulence}

Les caractétistiques de la couche limite atmosphérique sont principalement définies par le profil vertical de vitesse de vent sur les sites étudiés ainsi que par les conditions de

PROFILS VERTICAUX

DE VITESSES DE VENT

\section{ALTITUDE DE REFERENCE $Z=10 \mathrm{~m}$}

U $(\mathrm{m} / \mathrm{s})$

15

10

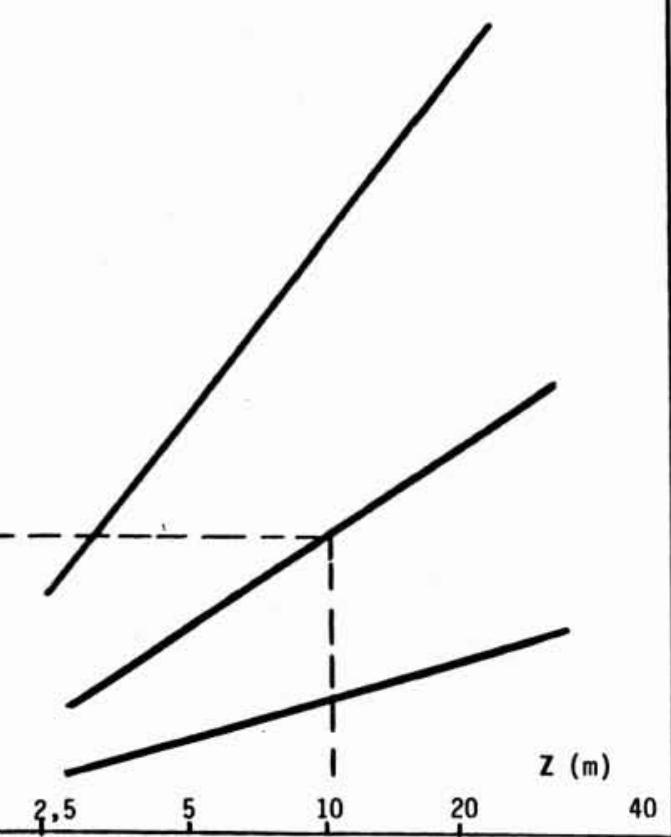

7. Profil vertical de vitesse du vent. 
turbulence atmosphérique dépendant des rugosités de surface.

S'il n'est pas indispensable de respecter une simulitude rigoureuse de Reynolds entre le modèle et la réalité, il convient par contre de vérifier que les écoulements y sont de même nature, c'est-à-dire turbulents, et de contrôler que dans la gamme de vitesses étudiée sur le modèle, la couche limite atmosphérique n'est pas affectée par la variation du nombre de Reynolds (profil de vitesse adimensionnalisé, concentration adimensionnalisée). La figure 7 donne un exemple des profils verticaux de vitesses de vent mesurés sur la maquette d'un site urbain, en bon accord avec la loi théorique de type :

$$
U_{(\mathrm{Z})} / U^{*}=(1 / K) \cdot \log _{\mathrm{e}}(\mathrm{Z} / \mathrm{Z} o)
$$

où $K$ est une constante, $Z o$ une hauteur fictive caractéristique du site et de l'ordre de $1 \mathrm{~m}$ pour un site urbain, $U^{*}$ la vitesse de frottement.

Les profils verticaux de vitesse de vent sont mesurés par anémométrie LASER fonctionnant sur le principe de l'effet DOPPLER. Ces procédés permettent également de mesurer et d'enregistrer les fluctuations instantanées de vitesses de l'écoulement et d'en déduire la turbulence moyenne au point considéré (exemple fig. 8 ), les résultats obtenus sont en bon accord avec les valeurs habituellement utilisées

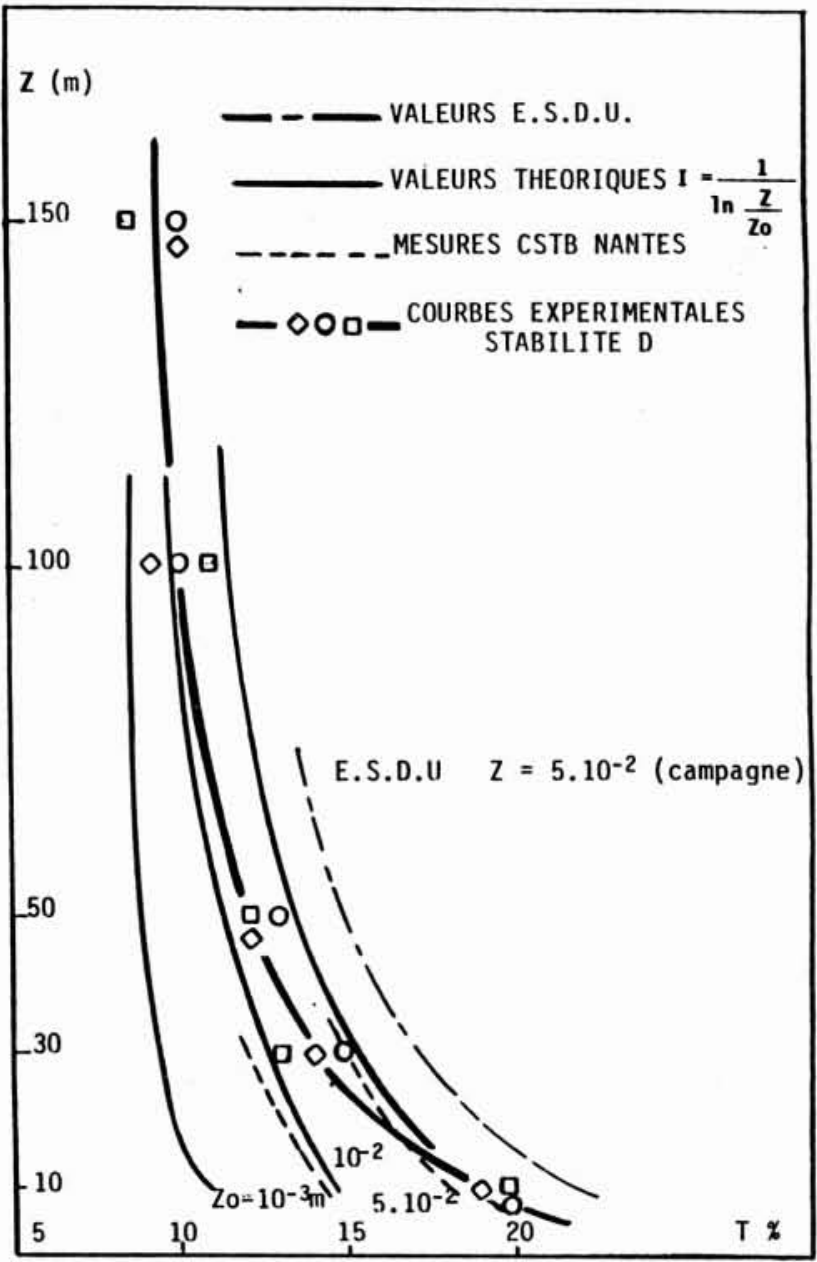

8. Profil vertical de turbulence atmosphérique. (théoriques, E.S.D.U., COUNIHAN, mesures du C.S.T.B.).

\subsubsection{Echelles de similitude}

Les échelles géométriques des maquettes couramment utilisées pour l'étude de la diffusion des polluants dans l'environnement sont de l'ordre du $1 / 30 \rightarrow 1 / 100$ (ventilation de bâtiments), $1 / 100 \rightarrow 1 / 300$ (étude dans le champ proche : cheminée, impact de tunnels routiers...), $1 / 200 \rightarrow 1 / 600$ pour les études de danger.

La modélisation de l'atmosphère et des rejets de polluants dans cette atmosphère est réalisée en respectant un certain nombre de conditions de similitude entre le modèle et la réalité $(t a b l .1)$ : similitude de FroudeRichardson, rapports de vitesses et de densités entre les effluents et l'atmosphère, ..., associés à des régimes de turbulence homologues caractérisés par les nombres de Reynolds représentatifs des phénomènes étudiés.

Tableau 1.

$\begin{array}{ll}U / g D^{1 / 2} & : \text { Similitude de FroudE } \\ (\Delta \rho / \rho) \cdot g D / U^{2} & : \text { Similitude de RICHARDSON } \\ U / V & : \text { Similitude du rapport des vitesses d'é- } \\ \Delta \rho / \rho & : \text { Simsion aux vitesses de vent } \\ V D / \nu & : \text { Reynolde caractéristique } \\ \text { où } U & : \text { Vitesse d'émission des gaz } \\ V & : \text { Vitesse du vent } \\ D & : \text { Dimension caractéristique } \\ \rho & : \text { Masse spécifique de l'air ambiant } \\ \Delta \rho & : \text { Ecart de masse spécifique des rejets, par } \\ g & : \text { rapport à l'air ambiant } \\ & \text { Accélération de la pesanteur }\end{array}$

\subsection{De nombreuses applications dans l'environnement}

\subsubsection{Etude d'impact de rejets urbains ou industriels}

L'utilisation de colorants (fluorescéine, rhodamine, autres...) permet de différencier l'impact de différentes sources polluantes, d'obtenir une très bonne visualisation de la trajectoire des panaches ainsi que des zones de retombées (fig. 9 et 10).

Les niveaux moyens de concentration dans les zones de retombées sont déterminés au moyen de prises d'échantillons et d'analyses colorimétriques ou conductimétriques. Ces valeurs, obtenues directement à l'échelle $1 / 1$ des taux de dilution sur la maquette du site étudié, sont alors comparées aux valeurs admissibles ou règlementaires pour juger de la conformité de l'installation ou des mesures compensatoires à prendre.

\subsubsection{Etude de ventilation de bâtiment et de captage de fumée pour dépoussiérage}

La figure 11 montre la maquette de l'installation de captage de fumée et poussières au-dessus d'un four électrique de 80 tonnes. 


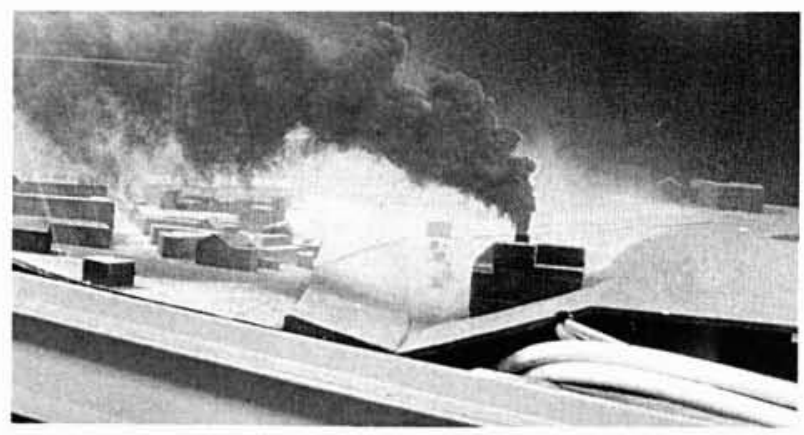

9. Etude d'impact sur l'environnement de cheminées (industrielle, urbaine...).

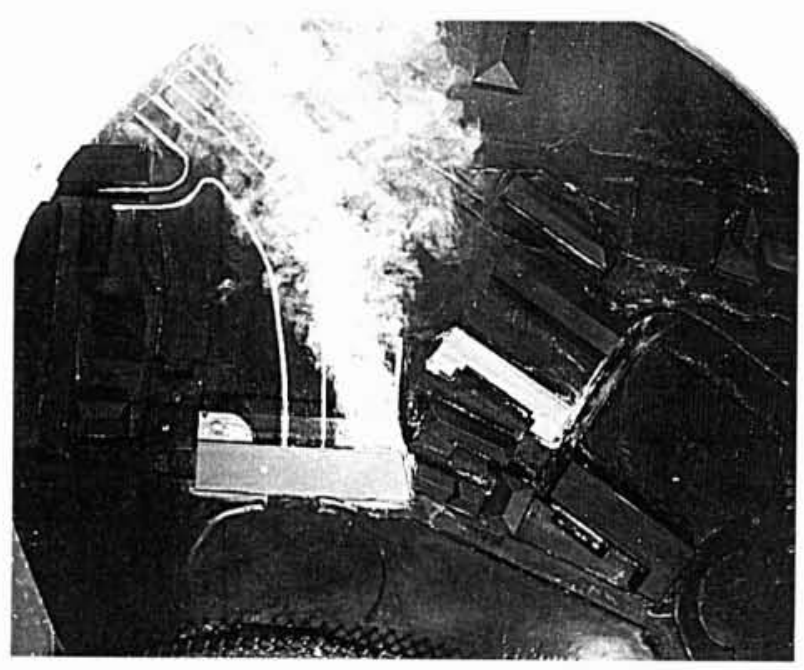

10. Etude d'impact de tunnel autoroutier.

L'étude permet d'optimiser l'emplacement, la forme, les dimensions de la hotte en toiture ainsi que les débits d'air à mettre en jeu qui peuvent, sur certaines installations actuelles, atteindre et quelquefois dépasser un million de $\mathrm{m}^{3} / \mathrm{h}$.

De telles études, qui doivent intégrer les contraintes de constructions, les tenues de toitures, le passage des ponts roulants, ont permis jusqu'à $30 \%$ de gain sur le débit de dépoussiérage conduisant à des économies appréciables en termes d'investissement et de coûts de fonctionnement.

\subsubsection{Etude de danger d'installations à risques}

Les études de dispersion des nuages de gaz toxiques ou inflammables dans l'atmosphère font souvent intervenir des gaz plus lourds que l'air avec propagation en nappe, associés à des considérations particulières d'ordre toxicologique liées à la sensibilité de l'organisme à ces polluants ou au danger induit (explosion, inflammation).

Ces études de diffusion en tunnel hydrodynamique sont réalisées dans le cadre des études de sécurité d'installations de production ou de stockage. Elles ont pour but d'analyser les conséquences sur l'environnement et sur l'individu de fuites accidentelles afin de déterminer les niveaux de
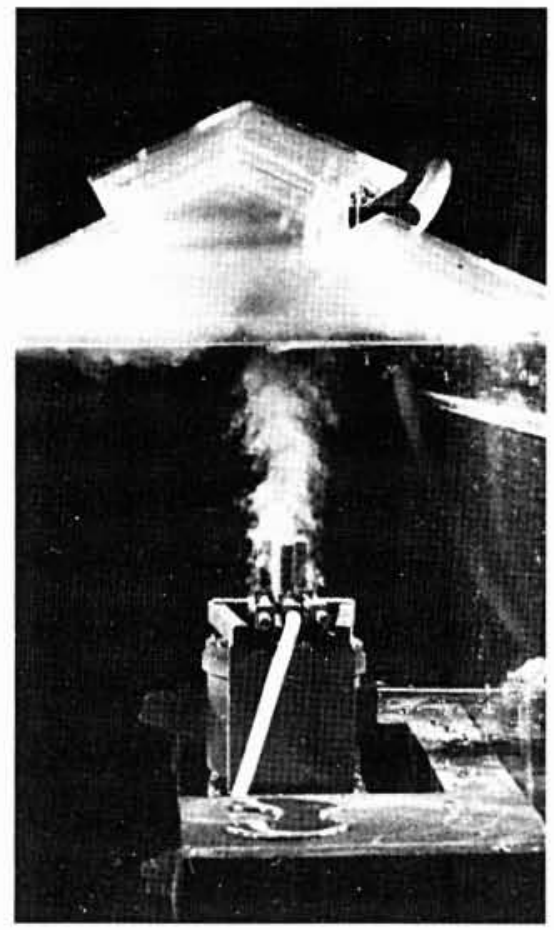

11. Optimisation sur maquette d'un captage de gaz et poussières.

risques atteints, les délais disponibles d'intervention et d'information des personnes, et de définir de possibles mesures de protection.

Les enregistrements réalisés en chaque point de mesure définissent à la fois les niveaux de concentrations atteints et les durées d'exposition au toxique au même point.

Les couples de valeurs " concentration-durée d'exposition » obtenus en différents points du site sont alors compa-

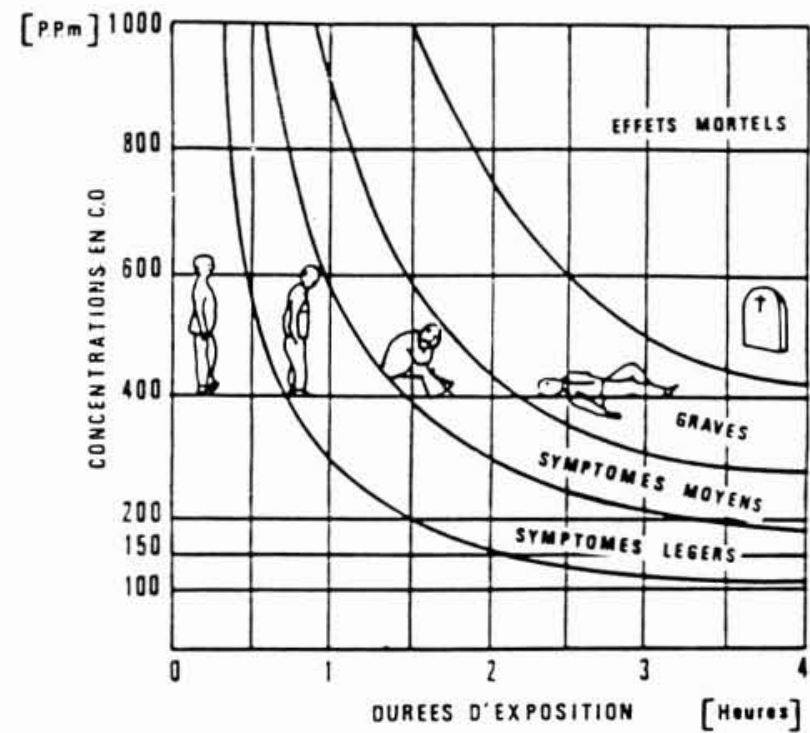

12. Relation dose-exposition du CO. 
rés aux couples de valeur « dose-effet " du gaz concerné (exemple fig. 12 pour le $\mathrm{CO}$ ), de façon à situer les risques encourus dans chacune des hypothèses d'accidents étudiées.

\section{Conclusions}

Nous n'évoquons là que deux exemples d'application parmi les multiples implications de la mécanique des fluides qui vont de l'étude des mélangeurs et des composants et circuits hydrauliques, aux études de pollution atmosphérique en passant par les coulées métallurgiques, l'étude de la cavitation, des bruits générés par les écoulements, etc...

Un certain nombre de réalisations industrielles consécutives à ces études ont permis de confirmer la validité des méthodes utilisées ainsi que des résultats obtenus dans les différents domaines d'application évoqués.

De très nombreuses études réalisées depuis plus d'une vingtaine d'années tant en France qu'à l'étranger permettent ainsi au Centre d'Etudes et de Recherches de Grenoble d'être particulièrement bien placé pour l'étude de tous ces problèmes de génie industriel et d'environnement auxquels les responsables techniques et d'aménagements publics et industriels sont de plus en plus sensibilisés. 